\title{
3D printing tablets: predicting printability and drug dissolution from rheological data
}

Moe Elbadawi $^{1}$, Thomas Gustaffson ${ }^{2}$, Simon Gaisford ${ }^{1,3}$, Abdul W. Basit ${ }^{1,3}$

${ }^{1}$ Department of Pharmaceutics, UCL School of Pharmacy, University College London, 29-39 Brunswick Square, London WC1N 1AX, UK.

${ }^{2}$ Control Engineering Group, Department of Computer Science, Electrical and Space Engineering, Luleå University of Technology, Luleå, Sweden

${ }^{3}$ FabRx Ltd., 3 Romney Road, Ashford, Kent, TN24 0RW, UK.

Corresponding author: m.elbadawi@ucl.ac.uk (M.Elbadawi), a.basit@ucl.ac.uk (A.W. Basit), s.gaisford@ucl.ac.uk (S.Gaisford) 


\begin{abstract}
Rheology is an indispensable tool for formulation development, which when harnessed, can both predict a material's performance and provide valuable insight regarding the material's macrostructure. However, rheological characterizations are under-utilized in 3D printing of drug formulations. In this study, viscosity measurements were used to establish a mathematical model for predicting the printability of fused deposition modelling 3D printed tablets (Printlets). The formulations were composed of polycaprolactone (PCL) with different amounts of ciprofloxacin and polyethylene glycol (PEG), and different molecular weights of PEG. With all printing parameters kept constant, both binary and ternary blends were found to extrude at nozzle temperatures of 130, 150 and 170 C. In contrast PCL was unextrudable at 130 and $150 \mathrm{C}$. Three standard rheological models were applied to the experimental viscosity measurements, which revealed an operating viscosity window of between 100-1000 Pa.s at the apparent shear rate of the nozzle. The drug profile of the printlets were experimentally measured over seven days. As a proof-of-concept, machine learning models were developed to predict the dissolution behaviour from the viscosity measurements. The machine learning models were discovered to accurately predict the dissolution profile, with the highest $f_{2}$ similarity score value of 90.9 recorded. Therefore, the study demonstrated that using only the viscosity measurements can be employed for the simultaneous high-throughput screening of formulations that are printable and with the desired release profile.
\end{abstract}

Keywords: Three-dimensional printing; 3D Printed drug products; Fused Deposition Modeling (FDM); Oral drug delivery systems; Artificial Intelligence; Machine Learning; Prediction Models.

\title{
1 Introduction
}

Fused deposition modelling (FDM) is a sub-class of three-dimensional printing (3DP) technologies and is a state-of-the-art fabrication technique in drug development (Aho et al., 2019; Goyanes et al., 2014; Trenfield et al., 2019a). FDM can print a range of drug delivery devices (DDS) that previously would have required multiple fabrication techniques (Beck et al., 2017; Eleftheriadis et al., 2019; Goyanes et al., 2016; Goyanes et al., 2015; Khaled et al., 2015; Tagami et al., 2019; Tagami et al., 2018; Trenfield et al., 2019b). Furthermore, it 
possesses both spatial and temporal resolution that make it highly desirable for both research and clinical applications (Kollamaram et al., 2018; Melocchi et al., 2019; Sadia et al., 2016; Smith et al., 2018). In addition, FDM printers demand a low laboratory footprint and are inexpensive. Such traits make FDM an ideal candidate technology to produce on-demand and personalized medicines (Verstraete et al., 2018), and in turn, improve patient quality of life. In order to realize this goal, several challenges will need to be addressed.

While promising in its ability to deliver novel DDS, other aspects of FDM are yet to be explored. The current approach to determining whether a formulation is printable via FDM employs a heuristic approach, where the feedstock for FDM is prepared under the assumption it can print. The feedstock for FDM are filaments, which are fabricated by hot-melt extruders (HME). Through the author's experience, a lot of filament is needed for testing a formulation's printability. However, this approach is both time- and resource-intensive. The problem is further compounded when researching expensive materials. If the prepared filament is found to be unusable, then it is destroyed. Hence, a more efficient approach is needed in order for FDM to be scaled for clinical use. The transition of FDM from laboratory to clinical settings would require the process to be automated and made intuitive for clinicians to use.

One characterization technique that captures the essence of FDM extrusion, and is a staple in formulation development, is rheology (Elbadawi et al., 2018; Isreb et al., 2019). Similar to FDM, recent rheometers can subject formulations to both heat and shearing, thereby mimicking the mode of actions exhibited in FDM. Rheological measurements are a multifaceted, where insight into polymer mechanical properties, stability, crystallization and degradation can be obtained (Aho et al., 2015; He et al., 2019; Madsen et al., 1998; Wu et al., 2006), to name a few. Previous work has shown that rheological data can be correlated to the performance of the material during fabrication (Shen et al., 2020). Another avenue of research has sought to use rheological data of feedstocks and correlate it to the properties of the final product (Höfl et al., 2006; Killion et al., 2011; Kim et al., 2018). This is because rheology can provide insight into material macrostructure (Calvet et al., 2004; Erwin et al., 2010). However, the application of rheology in 3DP of pharmaceuticals has not been thoroughly explored.

In comparison to the trial-and-error approach currently employed in FDM, rheology measurements require a small amount of material. In other 3DP techniques that are performed at room temperature, an intuitive understanding of the material's behaviour can be developed through visually inspecting the formulation (Gao et al., 2014; Gonzalez et al., 2017). However, 
FDM is a high-temperature technique, where the material's properties vary, and hence cannot be readily interpreted by examining the filaments in their cold state. Moreover, recent advances have seen in-line rheological sensors for real-time monitoring (Coogan and Kazmer, 2019, 2020), which have the potential to automate FDM printing, which is needed to translate the technology to clinical settings.

Although rheology provides a wealth of information about a material, the information can be overwhelming, particularly for inexperienced users. Researchers are afforded different rheological measurements to perform, from traditional viscosity measurements (Löbmann and Svagan, 2017) to the more advanced oscillatory amplitude shear studies performed for sensitive measurements (Salehiyan et al., 2014; Sun et al., 2011). As with large volumes of observations, it is challenging to discern patterns and would require years of experience. One technique that has seen a recent explosion in pattern recognition application is machine learning (ML), which uses cutting-edge models to discern patterns from complex datasets (Ekins et al., 2019; Liu et al., 2020). ML are a collection of algorithms capable of handling different formats of inputs, and can discern patterns in high-dimensional, non-linear datasets (LeCun et al., 2015; Singh et al., 2016). ML has revolutionized many medical industries, where it has successfully outperformed medical experts in their respective tasks (Nnamoko and Korkontzelos, 2020; Ting et al., 2018). ML has also been applied in drug formulation to predict processing conditions that would otherwise have required time and resources (Gentiluomo et al., 2020; Han et al., 2019; Lou et al., 2019). However, the use of ML in 3D printing pharmaceutics is thoroughly underdeveloped, with one study to date using ML for predicting FDM printing of tablets (Elbadawi et al., 2020).

This study sought to investigate the potential of rheology to predict the printability of formulations and to predict the drug release profile of the 3D printed formulations. A number of filaments were fabricated in-house using polycaprolactone, ciprofloxacin and different molecular weights of polyethylene glycol (PEG). All filaments were measured for their viscosity, which was correlated to their printing performance. In addition, machine learning models were developed to determine whether viscosity measurements can predict drug release. The study demonstrated that viscosity measurements could be used to screen materials for FDM printing, and with for the desired drug release profile. 


\section{Experimental Procedure}

\subsection{Starting Materials}

Polycaprolactone $(\mathrm{Mn}=80,000 \mathrm{~g} / \mathrm{mol})$, acetic acid (96\%), ciprofloxacin $(98.0 \%)$ and polyethylene glycol (PEG, Mw.: 4000 g.mol ${ }^{-1}$ and Mw.: 8000 g.mol ${ }^{-1}$ ) were purchased from Sigma-Aldrich Chemie GmbH (Steinheim, Germany, and St. Louis, U.S.A.). Polyethylene glycol (Mw.: 200 g.mol ${ }^{-1}$ ) was obtained from Fluka. Acetone ( $\geq 99.5 \%$ ) was purchased from VWR (France), and dichloromethane (DCM) were purchased from Fisher Scientific (Germany). All chemicals were used without further purification.

The reagents used for the dissolution study (HPLC and dissolution bath) were: Acetic Acid (Fluka, Steinheim, Germany), acetonitrile (LiChrosolv®, Merck, Darmstadt, Germany), methanol (Prepsolv®, Merck, Darmstadt, Germany), sodium hydroxide (puriss pa grade, Fluka, Steinheim, Germany) and potassium dihydrogen phosphate (purum grade, Fluka, Steinheim, Germany).

\subsection{Fabrication Process}

The raw materials were initially mixed with solvents to ensure a homogenous admixture is obtained, as previously detailed (Elbadawi, 2019). The solvent-casted films were then cut and loaded into a hot-melt extruder (Xcalibur, Noztek, UK. A torque speed of $20 \mathrm{rpm}$ was used, and the barrel temperatures were set to 120,100 and $60{ }^{\circ} \mathrm{C}$. The composition of the filaments and their sample name are provided in Table 1.

The filaments were used to fabricate the FDM prints. 3D models were generated using Solid Edge ST8 (Siemens PLM Software, Huntsville/AL, USA), with a diameter and height of 12 and $3 \mathrm{~mm}$, respectively. Subsequently, the .stl files were transferred to Cura 2.5 (Ultimaker B.V., The Netherlands) where the printing parameters were selected, and thereafter a g-code was generated.

The Ultimaker 3® (Ultimaker, Geldermalsen, The Netherlands) was used to 3D print the devices. The printing parameters were: a $0.4 \mathrm{~mm}$ nozzle temperature of $170{ }^{\circ} \mathrm{C}$; a print speed of $50 \mathrm{~mm} / \mathrm{s}$; a layer height of $0.25 \mathrm{~mm}$; infill density of $20 \%$; and a glass build plate temperature of $40{ }^{\circ} \mathrm{C}$. Fan cooling was enabled during the printing process, and the entire printing process took approximately 3 minutes per sample. Once the device was printed, it was left for two minutes prior to removing from the build-plate to prevent deformation by handling of the print. A representative image of a printlet is provided in the supplementary document (Figure S1). 
Commercial polylactic acid (PLA White, Ultimaker, Netherlands) and commercial PCL with a molecular weight of 50,000 da (Facilan ${ }^{\text {TM }}$ PCL 100, Elogi AM, Netherlands) were also used for comparison. Their recommended printing temperature as advised by their respective manufacturers were 200 and $120^{\circ} \mathrm{C}$, respectively. The final prints had a variation of $\pm 0.2 \mathrm{~mm}$ to the prescribed CAD model, which was determined by a Vernier caliper.

\subsection{Characterization Techniques}

\subsubsection{Differential Scanning Calorimetry (DSC)}

A Q1000 (TA Instruments, USA) DSC equipped with an autosampler, and nitrogen for both cooling and purging $(50 \mathrm{ml} / \mathrm{min})$ was used to determine the melting points of the admixtures. Bulk sample sizes of 5-8 mg were placed in hermetic aluminium pans with a pierced lid. The temperature was initially equilibrated to $-90{ }^{\circ} \mathrm{C}$, followed by a heating rate of $20^{\circ} \mathrm{C} / \mathrm{min}$ from -90 to $0{ }^{\circ} \mathrm{C}$, after which a heating rate of $5{ }^{\circ} \mathrm{C} / \mathrm{min}$ was employed until $275{ }^{\circ} \mathrm{C}$, which was the end of the run. The melting point was calculated as the onset of melting.

\subsubsection{X-Ray Diffractometry (XRD)}

An XRD Empyrean (Panalytical, Netherlands) was employed to examine the crystallinity of the bulk printed samples. Samples were scanned between 5 and $40^{\circ} 2$-theta values using a $\mathrm{CuK} \alpha$ source with a voltage of $45 \mathrm{kV}$ and a current of $20 \mathrm{~mA}$, and a step size of 0.026 , and 100 s per step.

\subsubsection{Attenuated Total Reflectance Fourier-Transformation Infra-red (ATR-FTIR)}

ATR-FTIR was performed using the vertex 80v (Bruker, Germany) in the mid-infrared region, equipped with platinum ATR accessory. 3D printed objects were directly placed onto the crystal, and the spectra were recorded at room temperature between 4000 and $400 \mathrm{~cm}^{-1}$, with a resolution of $4 \mathrm{~cm}^{-1}$. Prior to analyses, a background scan was performed. Tests were performed in triplicates.

\subsubsection{Rheological Measurements}

A temperature ramp to determine an ideal neat PCL nozzle extrusion temperature was performed. The test was conducted from 50 to $180{ }^{\circ} \mathrm{C}$ at a ramp rate of $5{ }^{\circ} \mathrm{C} / \mathrm{min}$ (i.e. similar to the DSC ramp rate), with a strain rate and angular frequency of $0.8 \%$ and $10 \mathrm{rad} / \mathrm{s}$, respectively. Time sweeps were also conducted, where the angular frequency used herein was $100 \mathrm{rad} / \mathrm{s}$ and a strain of $0.1 \%$. 
DHR 2 (TA Instruments, USA) was used to measure the viscosity of the samples. The filaments were cut into 10-20 mm lengths and placed on the Peltier plate, where the temperature was set to either 130,150 , or $170{ }^{\circ} \mathrm{C}$. An eight-millimetre parallel plate geometry with a gap of $0.4 \mathrm{~mm}$ was used for the viscosity measurements. An initial oscillatory amplitude sweep for neat PCL was performed to determine the linear viscoelastic region thereof. Accordingly, at a strain of $0.1 \%$, the measurements were conducted from 0.1 to $500 \mathrm{rad} / \mathrm{s}$. The steady-state shear was performed using the rheometers flow mode, and a time of 60 seconds was used for the measurements. For the Cox-Merz rule to upheld, the viscosity measurements were performed to obtain a discrepancy of less than $15 \%$. The apparent shear rate was determined as previously described (Elbadawi et al., 2018). All error bars represent the standard deviation of the data. All tests were performed in triplicates.

\subsubsection{Dissolution Study}

USP dissolution apparatus (Type II) was used to study the drug dissolution rate from the 3D printed objects. $900 \mathrm{~mL}$ of phosphate buffer $\mathrm{pH} 6.8$ was used as a dissolution medium. The experiments were performed at $37{ }^{\circ} \mathrm{C} \pm 0.5$, and the paddle was rotated at $50 \mathrm{rpm}$. Aliquot (6 $\mathrm{ml}$ ) of the samples was withdrawn from the vessel at different time points and replaced with a phosphate buffer of equal amount, for a period of seven days. The drug concentration was determined using high-performance liquid chromatography (HPLC) (Agilent 1200 infinity, Agilent Technologies, USA), in which a calibration curve was established. The HPLC protocol was followed in accordance with a previous study (Wu et al., 2008). All experiments were performed in triplicates due to sampling limitations. Dissolution was conducted under non-sink conditions and without the addition of surfactants in the media to mitigate the effect of polymer-surfactant interaction on the drug release behaviour. All error bars represent the standard deviation of the data. All tests were performed in triplicates.

\subsection{Machine Learning Models}

Machine learning models were developed in-house using Python v3.7 and using the scikit-learn package v0.21.3. The models used herein were multi-linear regression, decision tree and support vector machines. For the latter, the kernel function was set to linear. For each sample, the inputs used were the complex viscosity measurements obtained between 0.1 to $100 \mathrm{rad} / \mathrm{s}$ at $170{ }^{\circ} \mathrm{C}$. The output was the drug profile measured over the seven-day period. The models were trained on all but one sample, which was used as an unseen data to test the performance of the 
model. A python function was used to randomly split the data into training and testing, which selected sample 20CIP-10P8 as the test sample. No hyper-parameter tuning was employed for the ML models. For comparison, partial least square (PLS) analysis was fitted into the rheological data to predict the dissolution profile, using two components. PLS is a non-machine learning technique that is regularly used for developing prediction models. In this study, it serves as a baseline model to compare the effectiveness of the ML models.

For determining the efficacy of the models, the $f_{2}$ similarity factor was employed, for which the equation is:

$$
\boldsymbol{f}_{2}=50 \log _{10}\left\{\left[1+\frac{1}{n} \sum_{t=1}^{n} w_{t}\left(R_{t}-T_{t}\right)^{2}\right]^{-0.5} \times 100\right\}
$$

Where $n$ is the number of dissolution points, $R_{t}$ and $T_{t}$ are the measured and predicted dissolution predicted values, respectively, at time $t$. $w_{t}$ is the weighting factor, which was set to one.

\section{Results and Discussion}

PCL with different amounts of ciprofloxacin and PEG were successfully fashioned into filaments with the desired dimensions for FDM. The extrusion temperature examined were 130, 150 and $170{ }^{\circ} \mathrm{C}$, where $170{ }^{\circ} \mathrm{C}$ was used in previous research (Beck et al., 2017; Goyanes et al., 2016; Muwaffak et al., 2017), and formed the starting point for this study. The built-plate temperature was determined by a combination of differential scanning calorimetry and rheological measurements (Figure S2). The results revealed that during cooling, neat PCL solidified at approximately $40^{\circ} \mathrm{C}$. The DSC thermograph revealed that PCL began to crystallise at $30{ }^{\circ} \mathrm{C}$ (Figure S2 (c)). Rheological measurements, owing to its higher sensitivity, revealed that the loss modulus was the dominant moduli during cooling from $190{ }^{\circ} \mathrm{C}$ until $43{ }^{\circ} \mathrm{C}$, where a crossover was observed (Figure S2 (d)). Thereafter, the storage modulus dominated until 20 ${ }^{\circ} \mathrm{C}$. A storage modulus greater than the loss modulus is indicative of a viscoelastic material that is dominantly solid. The rheological measurements were able to detect the solidification process earlier than DSC owing to its higher measurement sensitivity. Accordingly, a temperature of $40{ }^{\circ} \mathrm{C}$ was selected to ensure that the layers exhibited sufficient mechanical stability to prevent collapsing under the above-deposited layers (Aho et al., 2019). 
Neat PCL was confirmed to print at $170{ }^{\circ} \mathrm{C}$; however, it did not extrude at the lower temperatures. Printing at $190{ }^{\circ} \mathrm{C}$ resulted in an improved flow of neat PCL, and consequently a better quality printlet. In contrast, all PCL blends of CIP and CIP with PEG were found to extrude at 130,150 and $170{ }^{\circ} \mathrm{C}$, and produced printlets. The only exception was 20CIP-15P2, which although extruded, it was unable to adhere to the platform. Increasing the temperature above $170{ }^{\circ} \mathrm{C}$ or decreasing below $130{ }^{\circ} \mathrm{C}$ had no effect, and the filament remained unprintable. The observations suggest that although CIP or CIP wit PEG were effective at lowering the nozzle temperature for printing PCL, not all compositions yielded a successful print. A previous study by the author found that incorporating ciprofloxacin or PEG altered the adhesion mechanism of PCL (Elbadawi, 2019). Following printing, x-ray diffraction and Fouriertransformation infrared spectroscopy were applied, which revealed that all components remained in the printlets (Figure $\mathbf{S 3}$ ).

\subsection{Predicting Printability using Rheological measurements}

Viscosity measurements of the filaments for 130 and $170{ }^{\circ} \mathrm{C}$ are presented in Figure 2. Before viscosity measurements, rheological time ramps were performed to ensure the stability of the materials as a function of time (Figure S4). The test was performed for five minutes, which was above the time it took for a viscosity measurement. The results confirmed that samples were rheologically stable for five minutes at both 130 and $170{ }^{\circ} \mathrm{C}$., and hence, any changes observed in viscosity (Figure 2) were as a function of shear rate.

The results are presented in both their steady- and dynamic-states. Viscosity measurements revealed that the addition of both 10 and $20 \mathrm{w} / \mathrm{w} \%$ of CIP reduced the initial plateau (also known as Newtonian plateau), which led to lower viscosity measurements across the measured shear range. The results verified that the addition of CIP plasticized PCL, and hence why it was printable at lower temperatures. The effect of PEG on PCL-CIP filaments varied depending on the PEG molecular weight and quantity thereof. Adding PEG 200 further reduced the viscosity of the polymer blend across all shearing range. Incorporating PEG 4000 or 8000 produced Newtonian plateau that were either comparable or greater than their respective PCL-CIP binary blends. This order was reversed at higher shear rates, where their viscosity was below their respective binary blends. This behaviour demonstrated that the incorporation of PEG 4000 or 8000 resulted in filaments that were more shear-thinning, and thus although presented with an initial higher viscosity, by the end of the test their viscosity 
was lower than their binary counterparts. Interestingly, differential scanning calorimetry tests performed on both filament and printlet samples did not reveal any plasticity effect from either ciprofloxacin or PEG (Figure S5), as a depression in melting point was not observed. Hence, it was concluded that rheological data was more sensitive to measuring plasticization of PCL by CIP and CIP with PEG.

The shearing events that occur in extrusion-based techniques occur at higher shear rates than what was feasible using the rotational rheometer (Aho et al., 2015; Suwardie et al., 2011). The apparent shear rate in the nozzle for this study was calculated to be $1000 \mathrm{~s}^{-1}$, which was in the same order of magnitude as the end of the test $\left(500 \mathrm{~s}^{-1}\right)$. To extrapolate the viscosity measurements to $1000 \mathrm{~s}^{-1}$, rheological models were fitted to the data, which are used to extrapolate the measured data to shear regions of interest (de Vicente et al., 2005; Frajkorová et al., 2016; Subhy, 2017; Wilson, 2019). The three models employed here were the Cross, Carreau-Yasuda and Williamson model, although the latter does not factor in an infinite shear region, but this was not necessary for this study. The red line fitted in Figure $\mathbf{2}$ is that of the Cross model $\left(\mathrm{R}^{2}>0.99\right)$, which is:

$$
\boldsymbol{\eta}=\boldsymbol{\eta}_{\infty}+\frac{\boldsymbol{\eta}_{\mathbf{0}}-\boldsymbol{\eta}_{\infty}}{\mathbf{1}+(\boldsymbol{k} \dot{\boldsymbol{\gamma}})^{\mathbf{1}-\boldsymbol{n}}}
$$

where $\eta$ is the viscosity, $\eta_{0}$ is the zero shear viscosity, $\eta_{\infty}$ is the infinite shear viscosity, $\dot{\gamma}$ is the shear rate, $k$ is the material consistency, and $n$ is the power-law index. The equations for the Williamson and Carreau-Yasuda model can be found in the supplementary document (Eq. (A1) \& (A2)). The derived viscosity at $\dot{\gamma}_{\text {app }}$ are enumerated in Table 2 , where the results extracted for both Carreau-Yasuda and Williamson are also presented.

All three models revealed that at the apparent shear rate, only PCL at $130 \mathrm{C}$ exhibited a viscosity in the order of $10^{3} \mathrm{~Pa} . \mathrm{s}$, whereas the other formulations were in the order of $10^{2}$ Pa.s. The PCL blends exhibited values in the order of $10^{2} \mathrm{~Pa}$.s at both 130 and $170{ }^{\circ} \mathrm{C}$, ranging between 291 to 965 Pa.s. This is in agreement with Anderegg et al. (2019) ${ }^{14}$ who also reported values in the order of $10^{2} \mathrm{~Pa}$.s via their in-situ approach. To further validate the models, the same methodology was applied to commercial polylactide (PLA) and polycaprolactone filaments. Both filaments were found to print at their respective manufacturers' recommended temperature. Fitting the rheological models revealed that they too exhibited viscosities in the order of $10^{2}$ at the apparent shear rate. Printing the commercial PLA at $170{ }^{\circ} \mathrm{C}, 30^{\circ} \mathrm{C}$ below 
the recommended temperature, was found not to extrude, and subsequent modelling revealed the viscosity at the apparent shear rate was in the order of $10^{3}$ Pa.s. Thus, an operating viscosity range was established using a semi-empirical model.

Very few studies have investigated the ideal viscosity for FDM printing (Azad et al., 2020). The proposed approach offers an alternative to using viscosity measurements to predict 3D printing. Ilyes et al. (2019) investigated a range of polymeric excipients, and suggested that FDM can work with complex viscosities up to $1200 \mathrm{~Pa} . \mathrm{s}$ at $100 \mathrm{rad} / \mathrm{s}$ are printable (Ilyés et al., 2019). The semi-empirical model developed herein is an extension of their work, which factors in the FDM parameters (i.e. printing speed and temperature). Coogan et al. (2019) developed an in-line rheometer that was inserted at the nozzle of their FDM printer, which was used to measure the viscosities of polycarbonate and high-impact polystyrene. The researchers explored different processing conditions and found that viscosities were in the order of $10^{2} \mathrm{~Pa} . \mathrm{s}$ for all but one of their 18 experiments (Coogan and Kazmer, 2019). However, in-line rheometers are not readily available and are complex to build.

\subsection{Predicting Dissolution using Rheology and Machine Learning}

The formulations that were printed were thereafter tested for their dissolution profile, over seven days (Figure 3). The binary blends containing PCL and CIP presented with the lowest release rate. As expected, the addition of PEG amplified the release rate, with samples containing PEG 200 were observed to release the most. Hence, the data is in concert with previous results that PEG can enhance drug release, and that different molecular weights result in different drug release profiles. The molecular weight of PEG influences the drug release because different molecular weights exhibit different swelling kinetics, and also dissolve at different rates (Paun et al., 2012). Furthermore, the dissolution of PEG induces in-situ pores, thereby exposing more of the delivery system surface area to the dissolution media. Different molecular weights of PEG will produce varying porous structures, which in turn will affect the drug release rate from the polymer matrix.

Predicting the dissolution profiles of the drug from their respective viscosity profiles was performed by splitting the results into eight samples for training, and holding one back to test the models' capabilities. The sample held back was, in essence, used to see how the machine learning models performed with unseen data. Four common machine learning models were used, which were multi-linear regression (MLR), decision trees and support vector 
machines (SVM). In addition, a non-machine learning predictive algorithm, partial least squares (PLS), was also used to determine whether the ML models can outperform a widelyused conventional algorithm.

The performance of the models is illustrated in Figure 4. All ML models were able to perfectly learn the training data, with a slight deviation observed in SVM for the two lower release profiles. PLS, on the other hand, was not as effective at learning the training data. Noticeable overestimates of release were observed for 10CIP and 20CIP-10P2. Once the models were built, they were then applied to the unseen sample, which was 20CIP-10P8. The $f_{2}$ similarity score is a standard measurement used to determine the effectiveness of prediction. It is a unitless value that measures the closeness between the predicted and actual profiles. The FDA has set a public value of 50-100 to indicate similarity between two dissolution profiles (Jasińska-Stroschein et al., 2017). It was applied in this study as a quantitative measurement for model accuracy, where it was discovered that decision tree obtained a considerably greater accuracy than the other models. An $f_{2}$ value of 90.9 was obtained, while the other three models obtained values between 50.2 to 57.5. Surprisingly, given its weak performance in learning the training data, PLS produced the second-highest $f_{2}$ score.

All four models were able to achieve a similarity score set by the FDA, and hence all models were acceptable in their predicting proficiency. Decision trees remarkable outperformance infers that the data is non-linear since PLS, MLR and SVM only discern linear relationships. Of great significance, this is the first study to use rheological measurements to predict dissolution profiles. Previous works have also used ML in their predictions. A recent study in $3 \mathrm{D}$ printing used ML to predict the dissolution profile using the formulation composition and one printing parameter as inputs (Madzarevic et al., 2019).

Two models were developed in the study, where one model obtained an $f_{2}$ score of 52.15, and the second obtained 44.91. The challenge of using a formulation's components and/or a printer's function is that it may not translate to other formulations. If new materials are to be used in the formulation, then the ML model would have to be trained again. Moreover, storage conditions and pre-treatments may affect material performance, which makes a model built on this approach susceptible to external factors. Using a fundamental property of a formulation, like viscosity, prevents subjectivity, and the model does not need to be re-trained when new materials or grades are used. A more conventional approach employed is to use vibrational spectroscopy data as inputs in developing ML models (Baranwal et al., 2019; 
Donoso and Ghaly, 2005; Pawar et al., 2016). Recently near-infrared spectroscopy and Raman spectroscopy were individually combined with ML to predict dissolution profiles, where the highest $f_{2}$ score reported was 90.53 (Galata et al., 2019). The challenge of using vibrational spectra is that the pre-processing of the data is required, which is laborious. The present study used the raw viscosity measurements and without pre-processing. One principal advantage of vibrational spectroscopies is that they can be non-destructive.

The salient phenomenon shared by both rheology and dissolution studies is the disentanglement of the polymer chains (Chakravorty et al., 2016; Wang, 2015). Previous work has reported that a change in rheological properties is consequently manifested in a change in dissolution profile (Bonferoni et al., 1992; Chakravorty et al., 2016). The present study builds on previous work by demonstrating that quantitative estimations of the drug release can be made using ML. It is the first to investigate the use of rheology and machine learning to predict drug dissolution profiles. Further work is needed to develop a more robust predictive model. The current approach will need to be tested on a larger dataset, comprising of different formulations. In addition, more sensitive rheological measurements should be incorporated when building the models, which can aid the ML models to discern nuanced changes in formulation.

\section{Conclusion}

The current study demonstrated the potential of rheology to predict both printability and dissolution profiles for formulations prepared for FDM printing. A semi-empirical model was developed to determine the ideal viscosity of filaments, which was found to be in the order of $10^{2} \mathrm{~Pa}$.s at the apparent shear rate used for printing. Filaments with a viscosity above $10^{3} \mathrm{~Pa} . \mathrm{s}$. were found to be unextrudable. The use of PEG were effective in reducing the printing temperature. However, the use of 15 w/w\% PEG 200 was found to unable to adhere to the build plate, which indicates that consideration should be given to the choice of PEG molecular weight. For predicting the dissolution profile, it was found that the non-linear model outperformed both ML linear models and PLS. An $f_{2}$ score of 90.9 was obtained for the testing data, which indicated a remarkable similarity between the predicted and actual dissolution profile. The current approach highlights the potential of using rheology to expedite the formulation stage of drug development. Future work will endeavour to produce a more robust predictive model for both printability and drug release profiles. 


\section{References}

Aho, J., Boetker, J.P., Baldursdottir, S., Rantanen, J., 2015. Rheology as a tool for evaluation of melt processability of innovative dosage forms. International Journal of Pharmaceutics 494, 623-642.

Aho, J., Bøtker, J.P., Genina, N., Edinger, M., Arnfast, L., Rantanen, J., 2019. Roadmap to 3DPrinted Oral Pharmaceutical Dosage Forms: Feedstock Filament Properties and Characterization for Fused Deposition Modeling. Journal of Pharmaceutical Sciences 108, 2635.

Azad, M.A., Olawuni, D., Kimbell, G., Badruddoza, A.Z.M., Hossain, M., Sultana, T., 2020. Polymers for Extrusion-Based 3D Printing of Pharmaceuticals: A Holistic Materials-Process Perspective. Pharmaceutics 12, 124.

Baranwal, Y., Román-Ospino, A.D., Keyvan, G., Ha, J.M., Hong, E.P., Muzzio, F.J., Ramachandran, R., 2019. Prediction of dissolution profiles by non-destructive NIR spectroscopy in bilayer tablets. International Journal of Pharmaceutics 565, 419-436.

Beck, R.C.R., Chaves, P.S., Goyanes, A., Vukosavljevic, B., Buanz, A., Windbergs, M., Basit, A.W., Gaisford, S., 2017. 3D printed tablets loaded with polymeric nanocapsules: An innovative approach to produce customized drug delivery systems. International Journal of Pharmaceutics 528, 268-279.

Bonferoni, M.C., Caramella, C., Sangalli, M.E., Conte, U., Hernandez, R.M., Pedraz, J.L., 1992. Rheological beheviour of hydrophilic polymers and drug release from erodible matrices. Journal of Controlled Release 18, 205-212.

Calvet, D., Wong, J.Y., Giasson, S., 2004. Rheological Monitoring of Polyacrylamide Gelation: Importance of Cross-Link Density and Temperature. Macromolecules 37, 77627771.

Chakravorty, A., Barman, G., Mukherjee, S., Sa, B., 2016. Effect of carboxymethylation on rheological and drug release characteristics of locust bean gum matrix tablets. Carbohydrate Polymers 144, 50-58.

Coogan, T.J., Kazmer, D.O., 2019. In-line rheological monitoring of fused deposition modeling. Journal of Rheology 63, 141-155.

Coogan, T.J., Kazmer, D.O., 2020. Prediction of interlayer strength in material extrusion additive manufacturing. Additive Manufacturing 35, 101368.

de Vicente, J., Stokes, J.R., Spikes, H.A., 2005. Lubrication properties of non-adsorbing polymer solutions in soft elastohydrodynamic (EHD) contacts. Tribology International 38, 515-526.

Donoso, M., Ghaly, E.S., 2005. Prediction of Drug Dissolution from Tablets Using NearInfrared Diffuse Reflectance Spectroscopy as a Nondestructive Method. Pharmaceutical Development and Technology 9, 247-263.

Ekins, S., Puhl, A.C., Zorn, K.M., Lane, T.R., Russo, D.P., Klein, J.J., Hickey, A.J., Clark, A.M., 2019. Exploiting machine learning for end-to-end drug discovery and development. Nature Materials 18, 435-441.

Elbadawi, M., 2019. Rheological and Mechanical Investigation into the Effect of Different Molecular Weight Poly(ethylene glycol)s on Polycaprolactone-Ciprofloxacin Filaments. ACS Omega 4, 5412-5423.

Elbadawi, M., Castro, B.M., Gavins, F., Ong, J., Gaisford, S., Perez, G., Basit, A.W., Cabalar, P., Goyanes, A., 2020. M3DISEEN: A Novel Machine Learning Approach for Predicting the 3D Printability of Medicines. International Journal of Pharmaceutics in press. 
Elbadawi, M., Rivera-Armenta, J., Cruz, B., 2018. Polymeric Additive Manufacturing: The Necessity and Utility of Rheology. Polymer Rheology 10.

Eleftheriadis, G.K., Ritzoulis, C., Bouropoulos, N., Tzetzis, D., Andreadis, D.A., Boetker, J., Rantanen, J., Fatouros, D.G., 2019. Unidirectional drug release from 3D printed mucoadhesive buccal films using FDM technology: In vitro and ex vivo evaluation. European Journal of Pharmaceutics and Biopharmaceutics 144, 180-192.

Erwin, B.M., Cloitre, M., Gauthier, M., Vlassopoulos, D., 2010. Dynamics and rheology of colloidal star polymers. Soft Matter 6, 2825-2833.

Frajkorová, F., Molero, E., Montero, P., Gomez-Guillen, M.C., Sanchez-Herencia, A.J., Ferrari, B., 2016. Biodegradable bi-layered coatings shaped by dipping of Ti films followed by the EPD of gelatin/hydroxyapatite composites. Journal of the European Ceramic Society 36, 343-355.

Galata, D.L., Farkas, A., Könyves, Z., Mészáros, L.A., Szabó, E., Csontos, I., Pálos, A., Marosi, G., Nagy, Z.K., Nagy, B., 2019. Fast, Spectroscopy-Based Prediction of In Vitro Dissolution Profile of Extended Release Tablets Using Artificial Neural Networks. Pharmaceutics 11, 400.

Gao, Y., Shi, W., Wang, W., Leng, Y., Zhao, Y., 2014. Inkjet Printing Patterns of Highly Conductive Pristine Graphene on Flexible Substrates. Industrial \& Engineering Chemistry Research 53, 16777-16784.

Gentiluomo, L., Roessner, D., Frieß, W., 2020. Application of machine learning to predict monomer retention of therapeutic proteins after long term storage. International Journal of Pharmaceutics 577, 119039.

Gonzalez, G., Chiappone, A., Roppolo, I., Fantino, E., Bertana, V., Perrucci, F., Scaltrito, L., Pirri, F., Sangermano, M., 2017. Development of 3D printable formulations containing CNT with enhanced electrical properties. Polymer 109, 246-253.

Goyanes, A., Buanz, A.B.M., Basit, A.W., Gaisford, S., 2014. Fused-filament 3D printing (3DP) for fabrication of tablets. International Journal of Pharmaceutics 476, 88-92.

Goyanes, A., Det-Amornrat, U., Wang, J., Basit, A.W., Gaisford, S., 2016. 3D scanning and 3D printing as innovative technologies for fabricating personalized topical drug delivery systems. Journal of Controlled Release 234, 41-48.

Goyanes, A., Wang, J., Buanz, A., Martínez-Pacheco, R., Telford, R., Gaisford, S., Basit, A.W., 2015. 3D Printing of Medicines: Engineering Novel Oral Devices with Unique Design and Drug Release Characteristics. Molecular Pharmaceutics 12, 4077-4084.

Han, R., Xiong, H., Ye, Z., Yang, Y., Huang, T., Jing, Q., Lu, J., Pan, H., Ren, F., Ouyang, D., 2019. Predicting physical stability of solid dispersions by machine learning techniques. Journal of Controlled Release 311-312, 16-25.

He, P., Yu, W., Zhou, C., 2019. Agglomeration of Crystals during Crystallization of Semicrystalline Polymers: A Suspension-Based Rheological Study. Macromolecules 52, 10421054.

Höfl, S., Kremer, F., Spiess, H.W., Wilhelm, M., Kahle, S., 2006. Effect of large amplitude oscillatory shear (LAOS) on the dielectric response of 1,4-cis-polyisoprene. Polymer 47, 72827288.

Ilyés, K., Kovács, N.K., Balogh, A., Borbás, E., Farkas, B., Casian, T., Marosi, G., Tomuță, I., Nagy, Z.K., 2019. The applicability of pharmaceutical polymeric blends for the fused deposition modelling (FDM) 3D technique: Material considerations-printability-process modulation, with consecutive effects on in vitro release, stability and degradation. European Journal of Pharmaceutical Sciences 129, 110-123.

Isreb, A., Baj, K., Wojsz, M., Isreb, M., Peak, M., Alhnan, M.A., 2019. 3D printed oral theophylline doses with innovative 'radiator-like' design: Impact of polyethylene oxide (PEO) molecular weight. International Journal of Pharmaceutics 564, 98-105. 
Jasińska-Stroschein, M., Kurczewska, U., Orszulak-Michalak, D., 2017. Statistical Considerations Concerning Dissimilar Regulatory Requirements for Dissolution Similarity Assessment. The Example of Immediate-Release Dosage Forms. Journal of Pharmaceutical Sciences 106, 1275-1284.

Khaled, S.A., Burley, J.C., Alexander, M.R., Yang, J., Roberts, C.J., 2015. 3D printing of fivein-one dose combination polypill with defined immediate and sustained release profiles. Journal of Controlled Release 217, 308-314.

Killion, J.A., Geever, L.M., Devine, D.M., Kennedy, J.E., Higginbotham, C.L., 2011. Mechanical properties and thermal behaviour of PEGDMA hydrogels for potential bone regeneration application. Journal of the Mechanical Behavior of Biomedical Materials 4, 12191227.

Kim, S.H., Yeon, Y.K., Lee, J.M., Chao, J.R., Lee, Y.J., Seo, Y.B., Sultan, M.T., Lee, O.J., Lee, J.S., Yoon, S.-i., Hong, I.-S., Khang, G., Lee, S.J., Yoo, J.J., Park, C.H., 2018. Precisely printable and biocompatible silk fibroin bioink for digital light processing $3 \mathrm{D}$ printing. Nature Communications 9, 1620.

Kollamaram, G., Croker, D.M., Walker, G.M., Goyanes, A., Basit, A.W., Gaisford, S., 2018. Low temperature fused deposition modeling (FDM) 3D printing of thermolabile drugs. International Journal of Pharmaceutics 545, 144-152.

LeCun, Y., Bengio, Y., Hinton, G., 2015. Deep learning. Nature 521, 436-444.

Liu, Q., Zhu, H., Liu, C., Jean, D., Huang, S.-M., ElZarrad, M.K., Blumenthal, G., Wang, Y., 2020. Application of Machine Learning in Drug Development and Regulation: Current Status and Future Potential. Clinical Pharmacology \& Therapeutics 107, 726-729.

Löbmann, K., Svagan, A.J., 2017. Cellulose nanofibers as excipient for the delivery of poorly soluble drugs. International Journal of Pharmaceutics 533, 285-297.

Lou, H., Chung, J.I., Kiang, Y.H., Xiao, L.-Y., Hageman, M.J., 2019. The application of machine learning algorithms in understanding the effect of core/shell technique on improving powder compactability. International Journal of Pharmaceutics 555, 368-379.

Madsen, F., Eberth, K., Smart, J.D., 1998. A rheological assessment of the nature of interactions between mucoadhesive polymers and a homogenised mucus gel. Biomaterials 19, 1083-1092.

Madzarevic, M., Medarevic, D., Vulovic, A., Sustersic, T., Djuris, J., Filipovic, N., Ibric, S., 2019. Optimization and prediction of ibuprofen release from 3D DLP printlets using artificial neural networks. Pharmaceutics 11, 544.

Melocchi, A., Uboldi, M., Inverardi, N., Briatico-Vangosa, F., Baldi, F., Pandini, S., Scalet, G., Auricchio, F., Cerea, M., Foppoli, A., Maroni, A., Zema, L., Gazzaniga, A., 2019. Expandable drug delivery system for gastric retention based on shape memory polymers: Development via 4D printing and extrusion. International Journal of Pharmaceutics 571, 118700.

Muwaffak, Z., Goyanes, A., Clark, V., Basit, A.W., Hilton, S.T., Gaisford, S., 2017. Patientspecific 3D scanned and 3D printed antimicrobial polycaprolactone wound dressings. International Journal of Pharmaceutics 527, 161-170.

Nnamoko, N., Korkontzelos, I., 2020. Efficient treatment of outliers and class imbalance for diabetes prediction. Artificial Intelligence in Medicine 104, 101815.

Paun, I.A., Moldovan, A., Luculescu, C.R., Staicu, A., Dinescu, M., 2012. MAPLE deposition of PLGA:PEG films for controlled drug delivery: Influence of PEG molecular weight. Applied Surface Science 258, 9302-9308.

Pawar, P., Wang, Y., Keyvan, G., Callegari, G., Cuitino, A., Muzzio, F., 2016. Enabling real time release testing by NIR prediction of dissolution of tablets made by continuous direct compression (CDC). International Journal of Pharmaceutics 512, 96-107. 
Sadia, M., Sośnicka, A., Arafat, B., Isreb, A., Ahmed, W., Kelarakis, A., Alhnan, M.A., 2016. Adaptation of pharmaceutical excipients to FDM 3D printing for the fabrication of patienttailored immediate release tablets. International Journal of Pharmaceutics 513, 659-668.

Salehiyan, R., Yoo, Y., Choi, W.J., Hyun, K., 2014. Characterization of Morphologies of Compatibilized Polypropylene/Polystyrene Blends with Nanoparticles via Nonlinear Rheological Properties from FT-Rheology. Macromolecules 47, 4066-4076.

Shen, Y., Tang, H., Huang, X., Hang, R., Zhang, X., Wang, Y., Yao, X., 2020. DLP printing photocurable chitosan to build bio-constructs for tissue engineering. Carbohydrate Polymers $235,115970$.

Singh, A., Thakur, N., Sharma, A., 2016. A review of supervised machine learning algorithms, 2016 3rd International Conference on Computing for Sustainable Global Development (INDIACom), pp. 1310-1315.

Smith, D.M., Kapoor, Y., Klinzing, G.R., Procopio, A.T., 2018. Pharmaceutical 3D printing: Design and qualification of a single step print and fill capsule. International Journal of Pharmaceutics 544, 21-30.

Subhy, A., 2017. Advanced analytical techniques in fatigue and rutting related characterisations of modified bitumen: Literature review. Construction and Building Materials $156,28-45$.

Sun, W., Yang, Y., Wang, T., Liu, X., Wang, C., Tong, Z., 2011. Large amplitude oscillatory shear rheology for nonlinear viscoelasticity in hectorite suspensions containing poly(ethylene glycol). Polymer 52, 1402-1409.

Suwardie, H., Wang, P., Todd, D.B., Panchal, V., Yang, M., Gogos, C.G., 2011. Rheological study of the mixture of acetaminophen and polyethylene oxide for hot-melt extrusion application. European Journal of Pharmaceutics and Biopharmaceutics 78, 506-512.

Tagami, T., Hayashi, N., Sakai, N., Ozeki, T., 2019. 3D printing of unique water-soluble polymer-based suppository shell for controlled drug release. International Journal of Pharmaceutics 568, 118494.

Tagami, T., Nagata, N., Hayashi, N., Ogawa, E., Fukushige, K., Sakai, N., Ozeki, T., 2018. Defined drug release from 3D-printed composite tablets consisting of drug-loaded polyvinylalcohol and a water-soluble or water-insoluble polymer filler. International Journal of Pharmaceutics 543, 361-367.

Ting, D.S.W., Liu, Y., Burlina, P., Xu, X., Bressler, N.M., Wong, T.Y., 2018. AI for medical imaging goes deep. Nature Medicine 24, 539-540.

Trenfield, S.J., Awad, A., Madla, C.M., Hatton, G.B., Firth, J., Goyanes, A., Gaisford, S., Basit, A.W., 2019a. Shaping the future: recent advances of 3D printing in drug delivery and healthcare. Expert Opinion on Drug Delivery 16, 1081-1094.

Trenfield, S.J., Xian Tan, H., Awad, A., Buanz, A., Gaisford, S., Basit, A.W., Goyanes, A., 2019b. Track-and-trace: Novel anti-counterfeit measures for 3D printed personalized drug products using smart material inks. International Journal of Pharmaceutics 567, 118443.

Verstraete, G., Samaro, A., Grymonpré, W., Vanhoorne, V., Van Snick, B., Boone, M.N., Hellemans, T., Van Hoorebeke, L., Remon, J.P., Vervaet, C., 2018. 3D printing of high drug loaded dosage forms using thermoplastic polyurethanes. International Journal of Pharmaceutics 536, 318-325.

Wang, S.-Q., 2015. Nonlinear rheology of entangled polymers at turning point. Soft Matter 11, 1454-1458.

Wilson, D.I., 2019. Industrial Applications of Yield Stress Fluids, in: Ovarlez, G., Hormozi, S. (Eds.), Lectures on Visco-Plastic Fluid Mechanics. Springer International Publishing, Cham, pp. 195-259.

Wu, D., Wu, L., Wu, L., Zhang, M., 2006. Rheology and thermal stability of polylactide/clay nanocomposites. Polymer Degradation and Stability 91, 3149-3155. 
Wu, S.-S., Chein, C.-Y., Wen, Y.-H., 2008. Analysis of ciprofloxacin by a simple highperformance liquid chromatography method. Journal of chromatographic science 46, 490-495.

\section{Table Captions}

Table 1. Sample name and composition of the HME filaments.

Table 2. The calculated viscosities (Pa.s) at the apparent shear rate determined by the Cross, Williamson, and Carreau-Yasuda rheological models. The table enumerates the results calculated at both 130 and $170{ }^{\circ} \mathrm{C}(\mathrm{R} 2>0.99$; the standard deviation of $\mathrm{n}=3)$.

\section{Figure Captions}

Figure 1. An overview of the present study: predicting printability and drug release using rheological measurements.

Figure 2. Viscosity Profiles of the filaments at 130 and $170{ }^{\circ} \mathrm{C}$. Both steady-state (open shapes) and dynamic-state (closed shapes) measurements are superimposed. The dynamic-state measurements were fitted with the Cross model, which is represented by the red line.

Figure 3. Dissolution profiles of the 3D printed tablets.

Figure 4. Dissolution profile prediction results when using viscosity measurements as inputs. The models were first built using nine samples (training data, left-hand side), and were evaluated by testing their ability to predict unseen data (right-hand side). $f_{2}$ score was used to quantitively determine the models accuracy (the cross and square data points represent the actual and predicted data, respectively). 